\section{EXATAS E TECNOLÓGICAS}

V.4 • N.1 2020 - Fluxo Contínuo

ISSN Digital: 2359-4942

ISSN Impresso: 2359-4934

DOI: 10.17564/2359-4942.2020v4n1p144-159
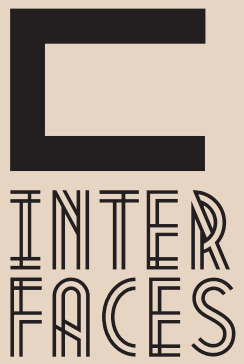

CIENTÍFICAS

\title{
TRANSPORTE DE CARGA ELÉTRICA NO SEMICONDUTOR 4H-SIC DOPADO TIPO P
}

TRANSPORT OF ELECTRIC LOAD IN HH-SIC DOPED TYPE SEMICONDUCTOR p

TRANSPORTE DE CARGA ELÉCTRICA EN SEMICONDUCTOR DE TIPO DOPADO 4H-SIC P

\section{RESUMO}

Neste trabalho foi realizado um estudo teórico sobre o transporte de carga no semicondutor carbeto de silício na forma $4 \mathrm{H}$-SiC dopado tipo $p$ e submetido a campos elétricos de baixa intensidade. $\mathrm{A}$ pesquisa foi realizada utilizando-se uma equação diferencial de movimento. A dependência do transporte de carga elétrica em função da intensidade e direção do campo elétrico aplicado e da temperatura foi analisada. Foi obtido que a maior mobilidade ocorre quando a direção do campo elétrico aplicado é perpendicular ao eixo cristalográfico $c$ do cristal semicondutor $4 \mathrm{H}-\mathrm{SiC}$ e que a mobilidade dos portadores sofre uma redução de aproximadamente $67 \%$ com o aumento da temperatura de 0 até $100^{\circ} \mathrm{C}$.

\section{PALAVRAS-CHAVE}

Semicondutores. Carbeto de Silício. Mobilidade. $4 \mathrm{H}-\mathrm{SiC}$. 


\section{ABSTRACT}

In this paper, a theoretical study was carried out on the charge transport in the semiconductor silicon carbide in the phase $4 \mathrm{H}-\mathrm{SiC}$ doped type $p$ and submitted to low intensity electric fields. The research was carried out using a differential equation of motion. The dependence of the electric charge transport according to the intensity and direction of the applied electric field and the temperature was analyzed. It was obtained that the greatest mobility occurs when the direction of the applied electric field is perpendicular to the crystallographic axis $\mathrm{c}$ of the $4 \mathrm{H}-\mathrm{SiC}$ semiconductor crystal, and that the hole mobility undergoes a reduction of approximately $33 \%$ with the increase in temperature from 0 to $100^{\circ} \mathrm{C}$.

\section{KEYWORDS}

Semiconductors. Silicon carbide. Mobility. 4H-SiC.

\section{RESUMEN}

En este trabajo se realizó un estudio teórico sobre el transporte de carga en el carburo de silicio semiconductor en forma de dopado $4 \mathrm{H}-\mathrm{SiC}$ tipo $p$ y sometido a campos eléctricos de baja intensidad. La investigación se llevó a cabo utilizando una ecuación diferencial de movimiento. Se analizó la dependencia del transporte de carga eléctrica en función de la intensidad y dirección del campo eléctrico aplicado y la temperatura. Se obtuvo que la mayor movilidad ocurre cuando la dirección del campo eléctrico aplicado es perpendicular al eje cristalográfico c del cristal semiconductor $4 \mathrm{H}-\mathrm{SiC}$, y esto experimenta una reducción de aproximadamente $67 \%$ con un aumento de temperatura de 0 a $100^{\circ} \mathrm{C}$.

\section{PALABRAS-CLAVE}

Semiconductores Carburo de silicio. Movilidad. 4H-SiC. 


\section{INTRODUÇ̧̃̃o}

Em relação à propriedade da condutividade elétrica os materiais podem ser agrupados em: isolantes, condutores, supercondutores e semicondutores. As características elétricas dos semicondutores estão situadas entre as características dos isolantes e as características dos condutores. Os semicondutores possuem resistividade elétrica no intervalo de $10^{-4}$ a $10^{7} \Omega$. m, que são valores intermediários entre os bons condutores $\left(10^{8} \Omega \cdot \mathrm{m}\right.$ ) e os bons isolantes (entre $10^{12}$ e $10^{20} \Omega \cdot \mathrm{m}$ ). As propriedades de um semicondutor dependem das impurezas, das excitações térmicas, dos defeitos da rede e dos desvios de suas composições químicas (KITELL, 1996).

Os elétrons de valência dos semicondutores formam pares covalentes, eles não são livres como no caso dos metais. Em temperaturas muito baixas, próximas do zero absoluto, em que as ligações covalentes se mantêm praticamente intactas, o cristal se comporta como um isolante. Entretanto, à medida que a temperatura cresce, algumas ligações covalentes se rompem, devido ao aumento do grau de agitação térmica. Em consequência, um dos elétrons ficou livre para vagar por todo o cristal, e ficou um "vazio" em uma das ligações covalentes do cristal.

Esse vazio é chamado de buraco. Os buracos também podem servir para o transporte de cargas. Quando uma ligação covalente está incompleta, isto é, quando existe um buraco, o elétron de um átomo vizinho pode deixar sua ligação covalente e vir preencher esse "vazio". Com isso, o elétron deixa um buraco em sua posição original, que por sua vez pode ser preenchido por outro elétron de uma ligação vizinha (KITELL, 1996).

No que se refere ao transporte de cargas, o buraco se comporta como uma carga positiva, com o mesmo valor absoluto da carga do elétron. Para considerar o buraco uma entidade real, é preciso lembrar que os buracos não passam de "vazios" nas ligações covalentes do cristal e destacar que o movimento dos elétrons e o movimento dos buracos se realizam de modo diferente: os elétrons deslocam-se no cristal de forma mais livre e os buracos só podem passar de ligação covalente para ligação covalente (KITELL, 1996).

Além da energia térmica, podemos controlar a densidade de portadores de carga mediante a adição de impurezas na rede cristalina. Essas impurezas são átomos com valência maior ou menor que o átomo que forma a rede cristalina do cristal semicondutor. O processo de adição de impurezas é denominado de dopagem. Os elétrons livres e buracos de um semicondutor são chamados de portadores, pois é por meio deles que se realiza o transporte de cargas elétricas de um ponto para outro do semicondutor. Os semicondutores dopados em que o transporte de carga é realizado pelos elétrons livres são chamados de "tipo n", e os semicondutores dopados em que o transporte de carga é realizado pelos buracos são chamados de "tipo p" (KITELL, 1996).

Os semicondutores de gap largo são particularmente atraentes para aplicações em dispositivos de alta potência e temperaturas operacionais elevadas (RODRIGUES, 2010). Dos semicondutores de gap largo candidatos para aplicações em dispositivos de alta potência, os mais atraentes são o GaN e também alguns dos vários politipos do SiC (TAKAHASHI, 2006). Em ambos materiais, a intensidade do campo elétrico de ruptura é cerca de quatro vezes maior que nos semicondutores Silício (Si) ou Arseneto de Gálio (GaAs). 
Além das propriedades similares ao GaN, como grande largura de gap, alta velocidade de saturação dos portadores e constante dielétrica pequena, o SiC tem uma condutividade térmica significativamente maior. Uma alta condutividade térmica é necessária para superar os efeitos de aquecimento do dispositivo, o que é particularmente importante em amplificadores de potência (WEITZEL, 1998).

O SiC pode se formar em muitas estruturas cristalinas distintas, as quais são denominadas de politipos (IZHEVSKYI, 2000), com alguns dos mais comuns, sendo o 3C-SiC (cúbico), o 4H-SiC (hexagonal) e o $6 \mathrm{H}-\mathrm{SiC}$ (hexagonal). Os politipos de $\mathrm{SiC}$ hexagonal possuem propriedades de transporte elétricas anisotrópicas, mas com grau e característica de anisotropia diferentes. A anisotropia está associada à simetria hexagonal, que faz com que as massas efetivas dos buracos difiram significativamente entre orientações paralelas ao eixo $c$ (que chamamos de $m_{\|}^{*}$ ), e aquelas ao longo do plano basal perpendicular ao eixo $c$ (que chamamos de $m_{\perp}^{*}$ ).

Entre os vários politipos de SiC, o 4H-SiC (FIGURA 1) foi reconhecido como o material mais atraente para operação em dispositivos eletrônicos de alta potência, alta frequência e alta temperatura devido a seu maior gap de energia e maior mobilidade de portadores que o de outros politipos (TAKAHASHI, 2006).

Figura 1 - Estrutura cristalina do $4 \mathrm{H}-\mathrm{SiC}$

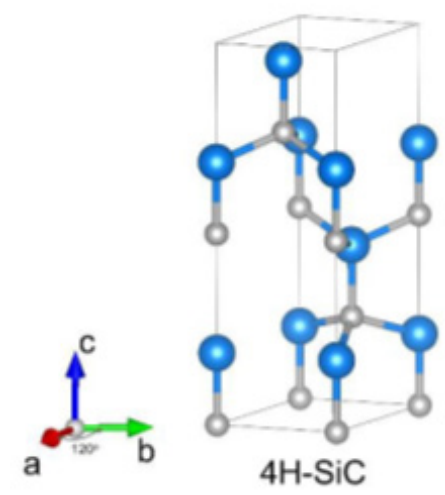

Fonte: Elaborado pelos autores.

Salientamos que para desenvolver dispositivos eletrônicos de alto desempenho, além de otimizar as etapas de fabricação, é necessário um bom conhecimento das propriedades de transporte. Como exemplo, a mobilidade de portadores é uma propriedade muito importante, afetando o desempenho do dispositivo. Dependendo do dispositivo em específico, a mobilidade dos portadores, será mais interessante ao longo do eixo c ou perpendicular ao mesmo. Em particular, a mobilidade de portadores ao longo do eixo c é extremamente importante em dispositivos de potência verticais como os diodos Schottky.

As propriedades ópticas e de transporte dos semicondutores tem sido estudadas usando Técnicas de Funções de Não-Equilíbrio de Green, Simulação de Monte Carlo, Equação de Balanço e Ensembles Estatísticos de Não-Equilibrado (KALOS, 2007; KALASHNIKOV, 1978; LUZZI, 2018; VAISSIERI, 1992). 
Aqui, utilizaremos a lei de força com adaptações quânticas para o estudo do transporte de portadores de carga positiva (buracos) no semicondutor $4 \mathrm{H}-\mathrm{SiC}$. $\mathrm{O}$ estudo de propriedades de transporte de semicondutores é extremamente importante do ponto de vista tecnológico, pois, as propriedades de transporte estão intimamente relacionadas com a eficiência dos dispositivos semicondutores.

Assim, o objetivo deste trabalho é estudar o transporte de portadores (buracos) no semicondutor $4 \mathrm{H}$-SiC quando o campo elétrico é aplicado na direção ao longo do eixo $c$ (o qual chamamos de $E_{\|}$), ou quando o campo elétrico $\vec{E}$ é aplicado na direção do plano perpendicular ao eixo $c$ (o qual chamamos de $E_{\perp}$ ) do cristal semicondutor. A velocidade de deriva dos buracos, o deslocamento, a mobilidade tanto no estado transiente quanto no estado estacionário foram obtidas e a dependência dessas propriedades com a intensidade e a direção do campo elétrico juntamente com a variação da temperatura foi analisada.

É extremamente importante do ponto de vista de aplicações em dispositivos eletrônicos comparar as propriedades de direções diferentes de um particular material. Uma direção pode ser mais conveniente que a outra em alguma aplicação tecnológica, sendo mais atrativa para certas aplicações em determinados dispositivos. Informamos que o estudo do transporte de carga elétrica no semicondutor carbeto de silicio nas formas $3 \mathrm{C}-\mathrm{SiC}$ e $6 \mathrm{H}-\mathrm{SiC}$ foram realizados e publicados recentemente (CORREA, 2019; FERRACIOLI, 2020).

\section{METODOLOGIA}

Consideraremos o semicondutor dopado tipo $p$ com uma concentraçãode impurezas menor que $10^{18} \mathrm{~cm}^{-3}$. Nesta condição não é necessário levar em consideração o espalhamento dos portadores de carga (buracos neste caso) pelas impurezas dopantes introduzidas na rede cristalina (RODRIGUES, 2009). O movimento dos portadores será governado por uma força externa aplicada e por uma força de resistência ao movimento . Para descrever tal situação utilizaremos uma equação semi-clássicabaseadanalei de força:

$$
\frac{d \vec{p}}{d t}=\sum \vec{F}=\vec{F}_{e x t}+\vec{f}
$$

Na Equação (1) aplicaremos a forma quântica do momento total e utilizaremos a expressão quântica para . A forma quântica do momento total é similar à clássica: , sendo a massa efetiva dos portadores. A massa efetiva dos portadores é uma massa que permite usar a segunda lei de Newton da Física Clássica. Isto porque a massa efetiva engloba os efeitos quânticos do potencial interno da rede cristalina sobre os portadores. Desta forma a definição de massa efetiva permite tratar os portadores como partículas clássicas de massa igual à sua massa efetiva. Assim, a Eq. (1) pode ser escrita como:

$$
m^{*} \frac{d \vec{v}(t)}{d t}=\vec{F}_{e x t}+\vec{f}
$$


A força externa $\vec{F}_{\text {ext }}$ será decorrente da aplicação de um campo elétrico. Escolhemos o campo elétrico $\vec{E}$ aplicado na direção do eixo. Assim, a força elétrica exercida sobre uma carga positiva é dada por:

$$
\vec{F}_{e l}=q \vec{E}=(+e)(+E \hat{\imath})=e E \hat{\imath} .
$$

Substituindo esta última expressão na Eq. (2), temos:

$$
m^{*} \frac{d \vec{v}(t)}{d t}=e E \hat{\imath}+\vec{f}
$$

Como o elétron está se movendo dentro do material semicondutor, há uma força de resistência $\vec{f}$ ao movimento do buraco, sendo considerada proporcional à velocidade $\vec{v}$ do buraco, ou seja: $\vec{f}=-\alpha \vec{v}$, , onde $\alpha$ é um parâmetro que está associado à resistividade elétrica do semicondutor (RODRIGUES, 2000). Utilizando esta expressão para $\vec{f}$ na Eq. (3), temos:

$$
m^{*} \frac{d v(t)}{d t}=e E-\alpha v(t)
$$

Notamos aqui uma semelhança da Equação (4) com a equação de Newton-Langevin (TOMÉ, 2001). A Equação (4) pode ser solucionada de forma exata por meio de técnicas de integração (RODRIGUES, 2017), obtendo-se:

$$
v(t)=\frac{e E}{\alpha}\left[1-e^{-\alpha t / m^{*}}\right]
$$

A equação anterior pode ser utilizada para determinar a equação da posição do buraco, lembrando que $v=\frac{d x}{d t}$. Após alguns cálculos obtemos:

$$
x(t)=\frac{e E}{\alpha}\left[t-\frac{m^{*}}{\alpha}\left(1-e^{-\alpha t / m^{*}}\right)\right]
$$

O parâmetro $\alpha$ que aparece nas Eqs. (5) e (6) está associado à resistividade elétrica do semicondutor, sendo dada por (RODRIGUES, 2000):

$$
\alpha=\frac{e^{2} \omega^{2} \gamma}{3} \sqrt{\frac{2 m^{* 3}}{\pi\left(k_{B} T\right)^{3}}} e^{z} \frac{2}{e^{2 z}-1} \mathrm{~K}_{1}(z)
$$

Onde eé a carga elementar do elétron $\left(1,602 \times 10^{-19} \mathrm{C}\right)$, wé a frequência de vibração da rede cristalina, $\mathrm{m}$ * é a massa efetiva dos buracos, $k_{\mathrm{B}}$ é a constante de Boltzmann cujo valor é aproximadamente $1,38 \times 10^{-16} \mathrm{erg} / \mathrm{K}, \mathrm{T}$ é a temperatura da rede cristalina, é a função modificada de segunda espécie de Bessel, e Ye zsão dados por: 


$$
\begin{aligned}
& \gamma=\frac{1}{s_{\infty}}-\frac{1}{s_{0}} \\
& z=\frac{(h / 2 \pi) \omega}{2 k_{B} T}
\end{aligned}
$$

Onde $E_{0}$ é a constante eletrostática estática, $E_{\infty}$ é a constante eletrostática de alta frequência, e $\mathrm{h}=6,626 \times 10^{-17} \mathrm{erg} . \mathrm{s}$ é a constante de Planck. A Tabela 1 mostra os principais parâmetros do semicondutor $4 \mathrm{H}$-SiC utilizados neste trabalho. Na Tabela 1 a quantidade $m_{0}$ é a massa de repouso do elétron $\left(m_{0}=9,109 \times 10^{-31} \mathrm{~kg}\right)$.

Tabela 1 - Parâmetros do semicondutor 4H-SiC

\begin{tabular}{|c|c|c|c|c|}
\hline$E_{0}$ & $E_{\infty}$ & $w(1014 \mathrm{~Hz})$ & $m^{*}\left(E_{\perp \_}\right)$ & $m *\left(E_{\|}\right)$ \\
\hline 10,0 & 6,7 & 1,82 & $0,59 m_{0}$ & $1,6 m_{0}$ \\
\hline
\end{tabular}

Fonte: (GÖTZ, 1993; PERSSON, 1997; IWATTA, 2001; BELLOTI, 2000).

\section{RESULTADOS}

Pode-se determinar, usando-se as Equações (5) e (6) e os dados contidos na Tabela 1 da seção anterior, a velocidade de deriva $v(\mathrm{t})$ e o deslocamento $x(\mathrm{t})$ dos portadores (buracos) em duas direções no semicondutor 4H-SiC: quando o campo elétrico é aplicado na direção paralela ao eixo $c\left(E_{\|}\right)$ou quando o campo elétrico é aplicado na direção perpendicular ao eixo $c\left(E_{\perp \_}\right)$. Aplicaremos este estudo para o caso do semicondutor $4 \mathrm{H}-\mathrm{SiC}$ dopado tipo $p$.

A Figura 2 apresenta a velocidade dos buracos em função do tempo para três valores de campo elétrico aplicados: $1 \mathrm{kV} / \mathrm{cm}, 2 \mathrm{kV} / \mathrm{cm}$ e $3 \mathrm{kV} / \mathrm{cm}$. A Figura 2(a) refere-se ao campo elétrico aplicado perpendicularmente ao eixo $c$ e a Figura 2(b) refere-se ao campo elétrico aplicado paralelo ao eixo cristalográfico $c$ do semicondutor. Analisando a Figura 2 nota-se que a velocidade do buraco com o campo elétrico aplicado paralelo ao eixo $c$ do semicondutor $4 \mathrm{H}-\mathrm{SiC}$ é menor que a velocidade do buraco com o campo elétrico aplicado perpendicular ao eixo $c$. Além disso, nos primeiros $0,5 \mathrm{ps}$, a velocidade cresce rapidamente (estado transiente) e, após 0,5 ps, se torna constante (estado estacionário). 
Figura 2 - Velocidade do buraco no estado transiente no 4H-SiC. A figura (a) refere-se ao campo elétrico aplicado perpendicularmente ao eixo $c$. A figura (b) refere-se ao campo elétrico aplicado paralelo ao eixo $c$
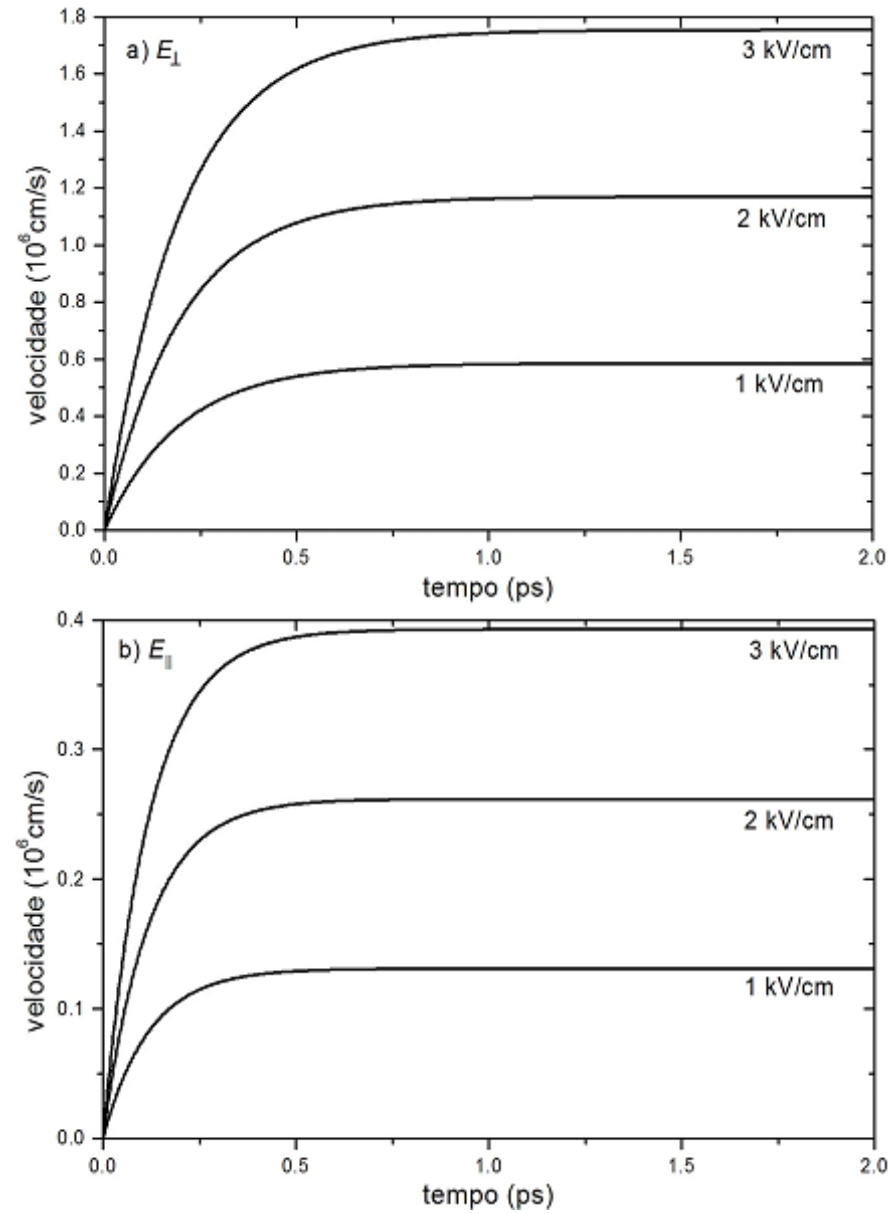

Fonte: Elaborado pelos autores.

Determina-se, utilizando a Eq. (6), a evolução temporal da posição do buraco no semicondutor $4 \mathrm{H}-\mathrm{SiC}$, em um intervalo de tempo de 3 ps, conforme ilustrado na Figura 3 para campos elétricos de $1 \mathrm{kV} / \mathrm{cm}, 2 \mathrm{kV} / \mathrm{cm}$ e $3 \mathrm{kV} / \mathrm{cm}$. A Figura $3(\mathrm{a})$ refere-se ao campo elétrico aplicado perpendicularmente ao eixo $c$ e a Figura $3(b)$ refere-se ao campo elétrico aplicado paralelo ao eixo $c$. Analisando a Figura 3 notamos que o buraco percorre uma distância maior no cristal semicondutor $4 \mathrm{H}-\mathrm{SiC}$ com o campo elétrico aplicado perpendicular ao eixo $c$. Essas informações possibilitam construir dispositivos com tamanhos específicos que podem levar ao dimensionamento mais eficiente dos custos de produção destes dispositivos. 
Figura 3 - Posição do buraco no semicondutor 4H-SiC. A figura (a) refere-se ao campo elétrico aplicado perpendicularmente ao eixo $c$. A figura (b) refere-se ao campo elétrico aplicado paralelo ao eixo $c$
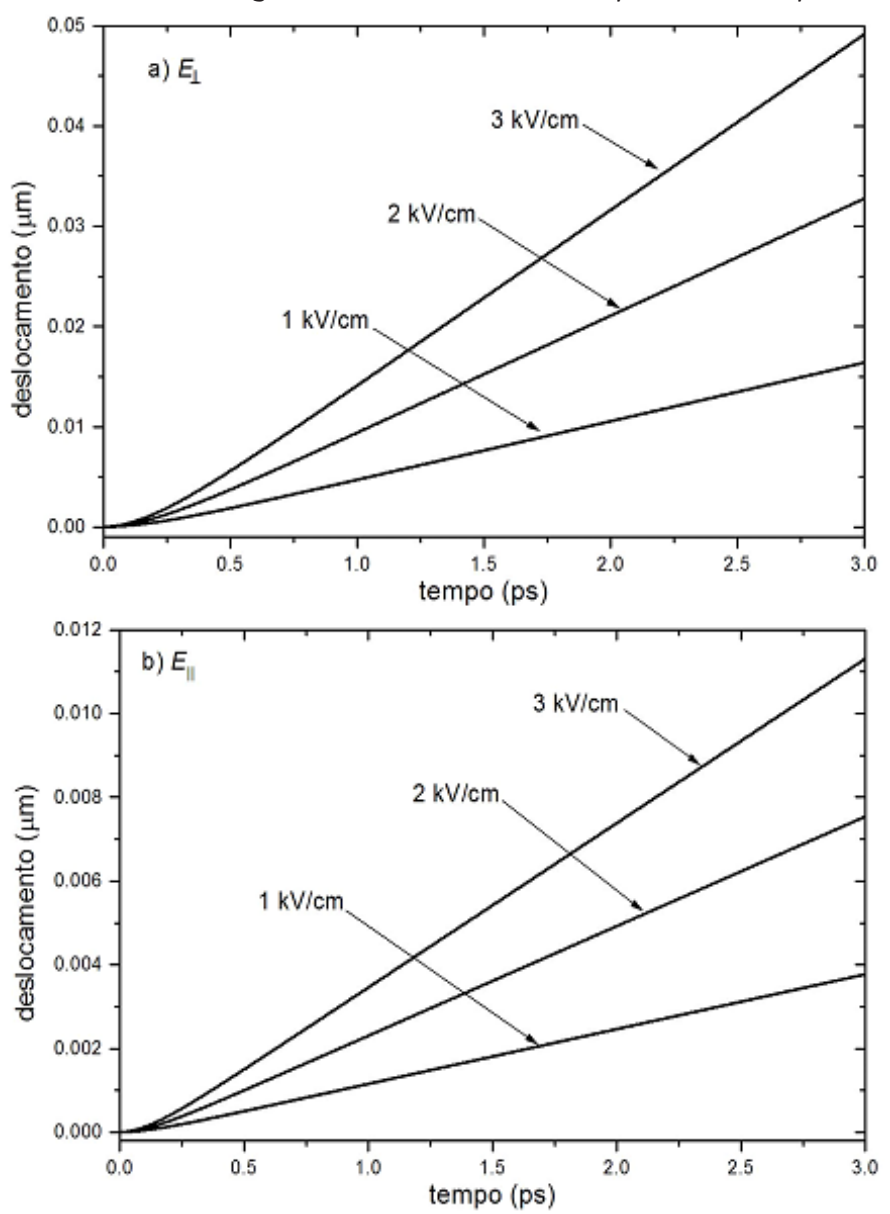

Fonte: Elaborado pelos autores.

Para um campo elétrico de $3 \mathrm{kV} / \mathrm{cm}$ a Figura 4 mostra em um único gráfico o deslocamento dos buracos para o campo elétrico aplicado perpendicular e paralelo ao eixo $c$. Nota-se que para $E_{\perp}$ os buracos percorrem uma distância de 49,15 nm em 3 ps enquanto que para $E_{\|}$uma distância de 11,30 $\mathrm{nm}$, isto é, com o campo elétrico aplicado perpendicularmente ao eixo $c$ a distância percorrida pelo buraco é aproximadamente 4,4 vezes a distância percorrida com o campo elétrico aplicado paralelo ao eixo $c$ neste intervalo de tempo de 3 ps. 
Figura 4 - Comparação do deslocamento dos buracos para um campo elétrico aplicado de $3 \mathrm{kV} / \mathrm{cm}$ (um aplicado perpendicular e outro aplicado paralelo ao eixo c)

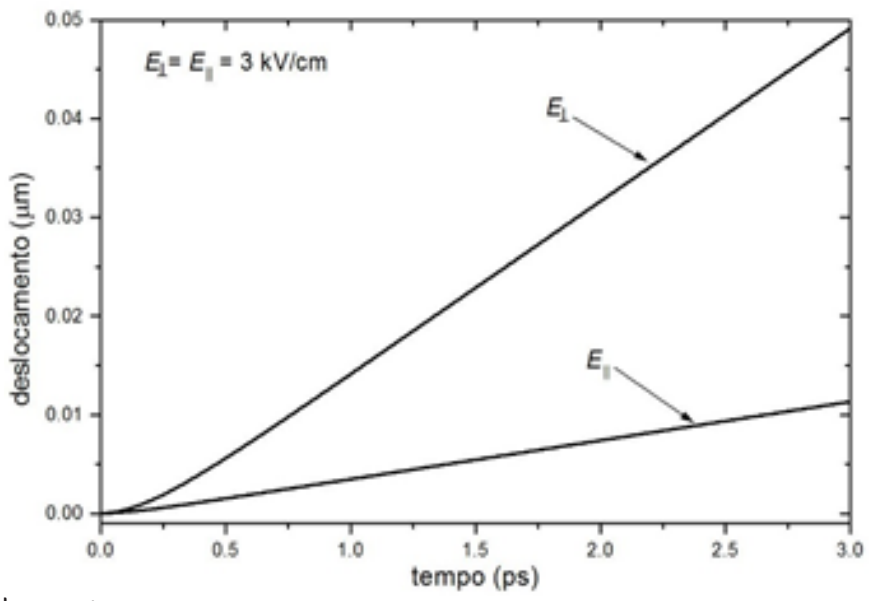

Fonte: Elaborado pelos autores.

O comportamento da velocidade de deriva do buraco no estado estacionário em função do campo elétrico aplicado de 0 a $3 \mathrm{kV} / \mathrm{cm}$ é apresentado na Figura 5. Observa-se que com o campo elétrico aplicado perpendicularmente ao eixo $c$ a velocidade do buraco é maior que com o campo elétrico aplicado paralelo ao eixo $c$, refletindo o comportamento do elétron no estado transitório, conforme a Figura 2. Nota-se também um comportamento linear da velocidade em relação ao campo elétrico aplicado, o que é característico do regime ôhmico, ocorrendo somente para baixos valores do campo elétrico aplicado, como é o presente caso.

Figura 5 - Velocidade do buraco em função da intensidade do campo elétrico

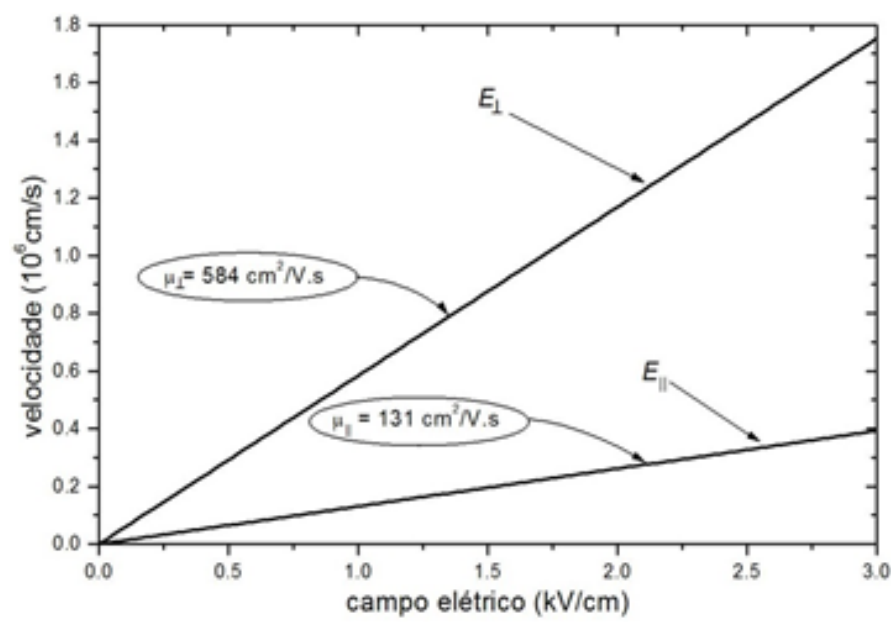

Fonte: Elaborado pelos autores. 
Uma importante propriedade de transporte dos semicondutores é a mobilidade elétrica $\mu$, definida como a velocidade de deriva dos portadores no estado estacionário dividida pelo valor do campo elétrico aplicado, ou seja, $\mu=v / E$ Assim no semicondutor $4 \mathrm{H}-\mathrm{SiC}$ temos dois valores para a mobilidade elétrica: uma quando o campo elétrico é aplicado paralelamente ao eixo cristalográfico $c$, $\mu_{\|}$, e outra quando o campo elétrico é aplicado perpendicularmente ao eixo cristalográfico $c, \mu_{\perp}$. Calculando-se a declividade das retas apresentadas na Figura 6 obtemos os seguintes valores: $\mu=584,3 \mathrm{~cm}^{2} /$ V.s e $\mu_{\|}=130,9 \mathrm{~cm}^{2} /$ V.s.

A Figura 6 ilustra o comportamento da velocidade estacionária dos buracos em função da temperatura da rede para as duas direções do campo elétrico aplicado no $4 \mathrm{H}$-SiC para campos elétricos aplicados com intensidades de $1 \mathrm{kV} / \mathrm{cm}, 2 \mathrm{kV} / \mathrm{cm}$ e $3 \mathrm{kV} / \mathrm{cm}$. Com o aumento da temperatura, aumenta-se a vibração da rede cristalina, aumentando a possibilidade de colisões dos portadores de carga (buracos) com os átomos da rede. Dessa forma, os buracos perdem parte de sua energia de movimento, fazendo com que a intensidade da velocidade seja reduzida.

Figura 6 - Velocidade estacionária dos buracos em função da temperatura da rede cristalina
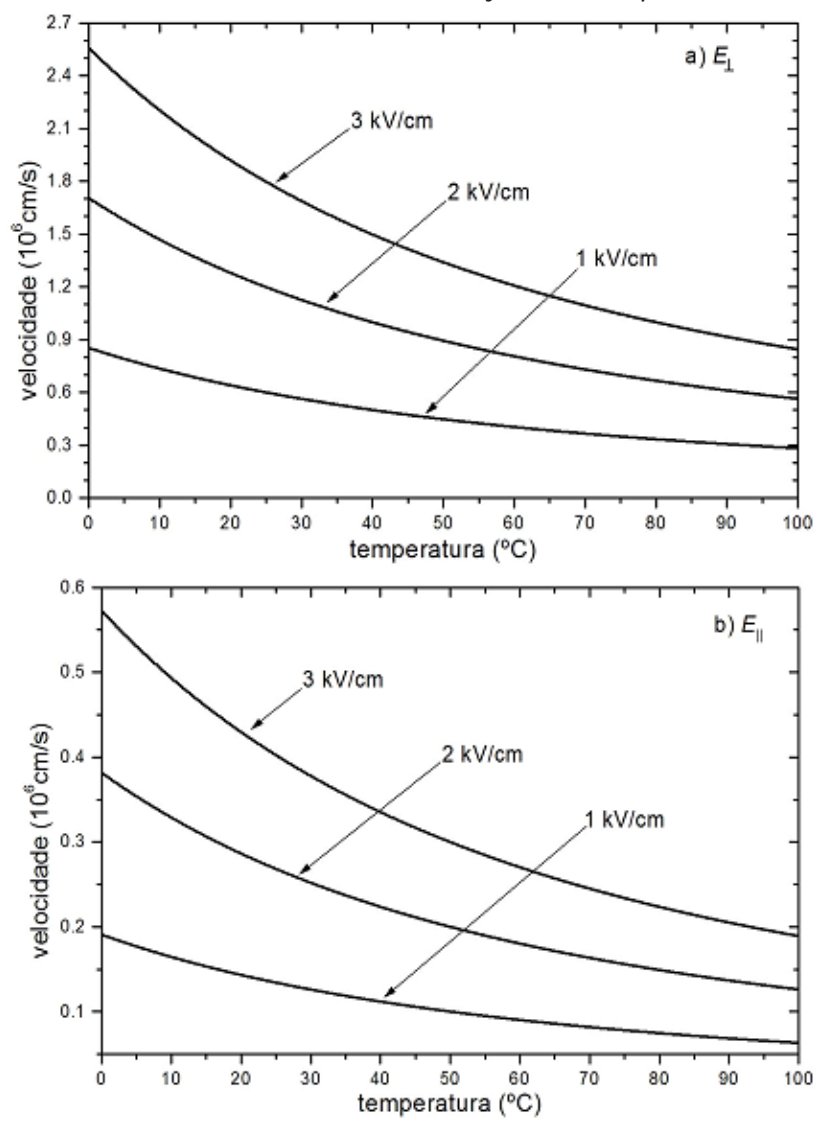

Fonte: Elaborado pelos autores. 
Para um campo elétrico fixo com intensidade de $3 \mathrm{kV} / \mathrm{cm}$ a Figura 7 mostra em um único gráfico a mobilidade dos buracos em função da temperatura para o campo elétrico aplicado perpendicular ou paralelo ao eixo $c$ do cristal semicondutor. Apesar da mobilidade não depender da intensidade do campo elétrico, nas condições aqui estudadas (baixos valores de campo elétrico) ela é severamente influenciada pela temperatura. De forma análoga à velocidade, a mobilidade dos buracos também diminui com o aumento da temperatura. A mobilidade tem uma redução de aproximadamente $67 \%$ de 0 a $100^{\circ} \mathrm{C}$.

Figura 7 - Redução da mobilidade eletrônica com o aumento da temperatura

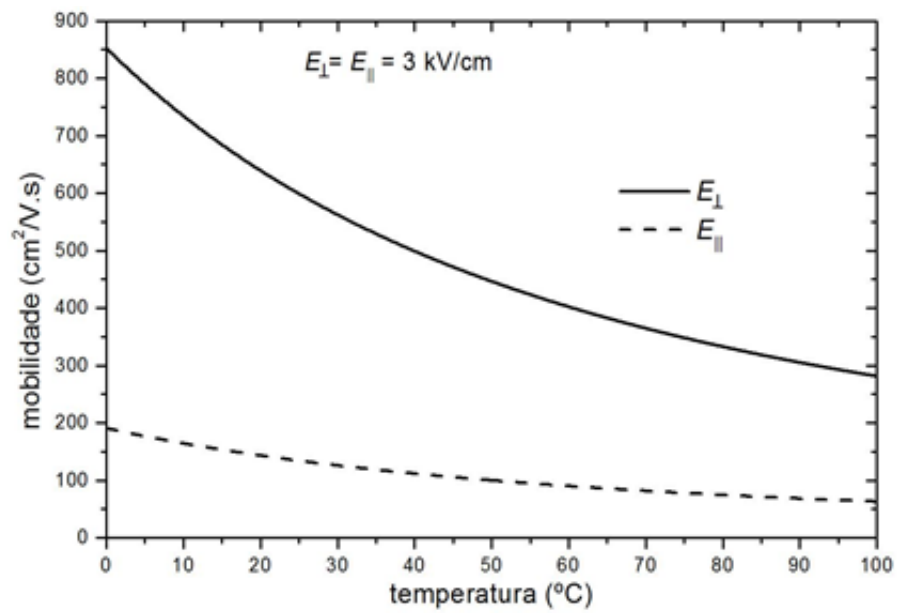

Fonte: Elaborado pelos autores.

A Tabela 2 mostra uma comparação de valores da mobilidade do semicondutor $4 \mathrm{H}-\mathrm{SiC}$ dopado tipo $p$ para uma temperatura de 300 kelvin e para baixos valores de campos elétricos aplicados (ou seja, em regime Ôhmico). Nota-se que o valor obtido neste trabalho aproxima-se do valor obtido por simulação computacional por Stefanakis et al. (2014) e um acordo que pode ser considerado razoável quanto ao valor experimental obtido por J. Pernot et al. (2001).

Enfatizamos que valores experimentais para a mobilidade variam muito de uma amostra para outra, variação esta que está intimamente relacionada com a qualidade e com as condições de crescimento do cristal. De acordo com F. Meng et al. (2019), devido à abundância de variados dados na literatura, tanto experimentais quanto teóricos, as propriedades elétricas de transporte do semicondutor $\mathrm{SiC}$ não têm sido muito bem conhecidas em nível microscópico. 
Tabela 2 - Valores da mobilidade do semicondutor 4H-SiC dopado tipo $\mathrm{p}$

\begin{tabular}{|c|c|c|}
\hline Referência & Método & Mobilidade (cm2/V.s) \\
\hline (STEFANAKIS, 2014) & Experimental & 96,6 \\
\hline (PERNOT, 2001) & Simulação & 123,6 \\
\hline Neste trabalho & Teórico & 130,9 \\
\hline
\end{tabular}

Fonte: Elaborado pelos autores.

\section{CONSIDERAÇÕES FINAIS}

Para o desenvolvimento de dispositivos de alta velocidade torna-se necessário conhecer em detalhes as propriedades dinâmicas dos elétrons e buracos nos semicondutores em escalas de tempo ultra-rápidas: da ordem de psicosegundos e de fentossegundos, dependendo do material semicondutor. A investigação de fenômenos ultra-rápidos em semicondutores é de grande interesse, uma vez que tem se tornado indispensável a utilização tecnológica de dispositivos que operem nessas condições ultra-rápidas como, por exemplo, detectores utilizados em aceleradores de partículas e circuitos que operam em sistemas de airbag em automóveis.

Neste trabalho determinamos teoricamente a velocidade de deriva, o deslocamento e a mobilidade de buracos no cristal semicondutor $4 \mathrm{H}-\mathrm{SiC}$ dopado tipo $p$, submetido a campos elétricos de baixa intensidade aplicados perpendicularmente ou paralelamente ao eixo cristalográfico $c$ do cristal semicondutor. Para tanto utilizamos uma equação semi-clássica baseada na segunda lei de Newton a qual conduz a uma equação de evolução do tipo Newton-Langevin.

Foi verificado que a maior velocidade de deriva dos buracos e consequentemente a maior mobilidade dos buracos ocorre quando o campo elétrico é aplicado perpendicularmente ao eixo $c$. Foi também determinada a variação da velocidade e da mobilidade em função da temperatura da rede, observando-se que ambas sofrem uma redução de aproximadamente $67 \%$, com o aumento da temperatura de 0 até $100^{\circ} \mathrm{C}$. Esta diminuição da mobilidade com o aumento da temperatura diminui a eficiência de dispositivos semicondutores. 


\section{REFERÊNCIAS}

BELLOTTI, E.; NILSSON, H. E.; BRENNAN, K. F.; RUDEN, P. P.; TREW, R. Monte Carlo calculation of hole initiated impact ionization in $4 \mathrm{H}$ phase SiC. Journal of Applied Physics, v. 87, n. 8, p. 3864$3871,2000$.

CORREA, A. M. D.; RODRIGUES, C. G.; LUZZI, R. Electron transport in bulk n-doped 3C-SiC by using a non-equilibrium quantum kinetic theory. European Physical Journal B, v. 92, p. 261, 2019.

FERRACIOLI, R. T.; RODRIGUES, C. G.; LUZZI, R. Anisotropic carrier transport in n-doped 6H-SiC. Physics of The Solid State, v. 62, p. 110-115, 2020.

GÖTZ, W.; SCHÖNER, A.; PENSL, G.; SUTTROP, W.; CHOYKE, W. J.; STEIN, R.; LEIBENZEDER, S. Nitrogen donors in 4H-silicon carbide. Journal of Applied Physics, v. 73, p. 3332-3338, 1993.

IWATAA, H.; ITOHB, K. M. Donor and acceptor concentration dependence of the electron Hall mobility and the Hall scattering factor in n-type $4 \mathrm{H}$ - and 6H-SiC. Journal of Applied Physics, v. 89, p. 6228-6234, 2001.

IZHEVSKYI, V. A.; GENOVA, L. A.; BRESSIANI, J. C.; BRESSIANI, A. H. A. Review article: silicon carbide. Structure, properties and processing. Cerâmica, v. 46, p. 297, 2000.

KALASHNIKOV, P. Equations of motion, Green's functions, and thermodynamic relations in theories of linear relaxation. Theoretical and Mathematical Physics, v. 35, p. 362-370, 1978.

KALOS; M. H.; WHITLOCK, P. A. Monte Carlo methods. New York: Wiley-Interscience, 2007.

KITELL, C. Introduction to solid state shysics, 7. ed. New York: John Wiley e Sons, 1996.

LUZZI, R.; VACONCELLOS, A. R.; RAMOS, J. G.; RODRIGUES, C. G. Statistical Irreversible Thermodynamics in the Framework of Zubarev's Nonequilibrium Statistical Operator Method. Theoretical and Mathematical Physics, v. 194, p. 4-29, 2018.

MENG, F.; MA, J.; HE, J.; LI, W. Phonon-limited carrier mobility and temperature-dependent scattering mechanism of 3C-SiC from first principles. Physical Review B, v. 99, p. 045201, 2019.

PERNOT, J.; ZAWADZKI, W.; CONTRERAS, S. ; ROBERT, J. L.; NEYRET, E.; CIOCCIO, L. Electrical transport in 4H silicon carbide. Journal of Applied Physics, v. 90, p. 1869-1878, 2001. 
PERSSON, C.; LINDEFELT, U. Relativistic band structure calculation of cubic and hexagonal SiC polytypes. Journal of Applied Physics, v. 82, n. 11, p. 5496-5508, 1997.

RODRIGUES, C. G. Influence of the concentration, temperature and electric field intensity on the electron mobility in n-doped zinc sulphide. European Physical Journal B, v. 72, p. 405-408, 2009.

RODRIGUES, C. G.; SILVA, C. A. B. ; VACONCELLOS, A. R.; RAMOS, J. G.; LUZZI, R. The role of nonequilibrium thermo-mechanical statistics in modern technologies and industrial processes: an overview. Brazilian Journal of Physics, v. 40, p. 63-91, 2010.

RODRIGUES, C. G. Tópicos de física matemática para licenciatura. São Paulo: editora LF, 2017.

RODRIGUES, C. G.; LUZZI, R. Semiconductor nonequilibrium statistics: applications to far-from equilibrium situations. Bristol: IOP Publishing Ltda., 2020.

STEFANAKIS, D.; ZEKENTES, K. TCAD models of the temperature and oping dependence of the bandgap and low field carrier mobility in 4H-SiC. Microelectronic Engineering, v. 116, p. 65-71, 2014.

TAKAHASHI, K.; YOSHIKAWA, A.; SANDHU, A. Wide bandgap semiconductors. Berlin: Springer, 2006.

TOMÉ, T.; OLIVEIRA, M. J. Dinâmica estocástica e irreversibilidade. São Paulo: USP, 2001.

VAISSIERE, J. C.; NOUGIER, J. P.; FADEL, M.; HLOU, L.; KOCEVAR, P. Numerical solution of coupled steady-state hot-phonon-hot-electron Boltzmann equations in InP. Physical Review B, v. 46, n. 20, p. 13082, 1992.

WEITZEL, C.; POND, L.; MOORE, K.; BHATNAGAR, M. Effect of device temperature on RF FET power density. Materials Science Forum, v. 264, p. 969, 1998. 


\section{(@) (1) (-)}

Este artigo é licenciado na modalidade acesso abertosob a Atribuição-Compartilhalgual CC BY-SA

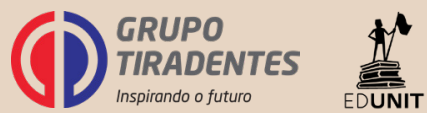

